Abanico Veterinario. Enero-Diciembre 2021; 11:1-13. http://dx.doi.org/10.21929/abavet2021.24

Artículo Original. Recibido: 21/01/2021. Aceptado: 28/04/2021. Publicado: 15/05/2021. Clave: e2021-01.

\title{
Calidad fermentativa y producción de metano en ensilados de rastrojo de maíz adicionados con nopal fermentado y sin fermentar
}

Fermentative quality and methane production in corn stover silage with fermented and unfermented nopal cactus

\section{Araiza-Rosales Elia ${ }^{1 \text { ID }}$, González-Arreola Adolfo ${ }^{\text {ID }}$, Pámanes-Carrasco Gerardo ${ }^{1 \text { ID }}$, Murillo-Ortiz Manuel $^{2}$ ID , Jiménez-Ocampo Rafael ${ }^{3}$ ID, Herrera-Torres Esperanza ${ }^{\star 4}$ ID}

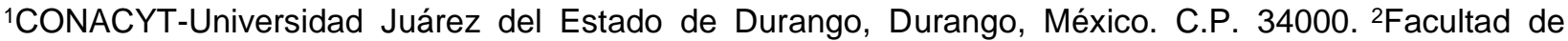
Medicina Veterinaria y Zootecnia, Universidad Juárez del Estado de Durango. Durango, México. ${ }^{3}$ Instituto Nacional de Investigaciones Forestales, Agrícolas y Pecuarias-Durango. Km 4.5 Carretera DurangoMezquital. Durango, México. ${ }^{4}$ Instituto Tecnológico del Valle del Guadiana-Tecnológico Nacional de México. Durango, México. e_araiza2002@hotmail.com, junior.glz@hotmail.com, gerardo.pamanes@gmail.com,_manuelmurilo906@gmail.com,_rafax77@hotmail.com, hetoes99@yahoo.com.mx

\section{RESUMEN}

El objetivo de esta investigación fue evaluar la calidad nutritiva, fermentativa y la emisión de metano en ensilajes de rastrojo de maíz con nopal (Opuntia ficus-indica), para lo cual se evaluaron tres tratamientos experimentales, T1: forraje de maíz; T2: $75 \%$ rastrojo de maíz + $25 \%$ nopal y T3: $75 \%$ rastrojo de maíz + $25 \%$ nopal fermentado. Se elaboraron 21 micro-silos (7 por tratamiento) en recipientes de plástico y se dejaron fermentar por 30 días. Al término de la fermentación se evaluó la composición química, los parámetros de fermentación, producción de gas y metano $\left(\mathrm{CH}_{4}\right)$. Los contenidos de materia seca (MS), proteína cruda (PC), fibra detergente neutro (FDN) y fibra detergente ácida (FDA) fueron diferentes entre los tratamientos $(p<0.05)$; el contenido de PC incrementó $44 \%$ al adicionar nopal fermentado (T3). Los valores de nitrógeno amoniacal, ácido láctico y ácidos grasos volátiles fueron diferentes entre tratamientos $(p<0.05)$. La máxima producción de gas (Gmax) y la concentración de $\mathrm{CH}_{4}$ disminuyeron $32 \%$ y 49 \% en T3, respectivamente. La adición de nopal y nopal fermentado a ensilados con rastrojo de maíz incrementa el contenido de proteína. Además, reduce la síntesis de metano ruminal in vitro.

Palabras clave: fermentación, rastrojo de maíz, gases efecto invernadero, ensilaje.

\begin{abstract}
The objetive of this research was to evaluate the nutritive and fermentative quality and methane emissions in corn stover silages with nopal (Opuntia ficus-indica), for which three experimental treatments were evaluated, T1: corn fodder; T2: $75 \%$ corn stover $+25 \%$ nopal; and T3: $75 \%$ corn stover $+25 \%$ fermented nopal. Twenty-one micro-silos (7 per treatment) were prepared in plastic containers and left to ferment for 30 days. At the end of fermentation, the chemical composition, fermentation parameters, gas production and methane $(\mathrm{CH} 4)$ were evaluated. Dry matter (DM), crude protein (CP), neutral detergent fiber (NDF) and acid detergent fiber (ADF) contents were different among treatments $(p<0.05)$; CP content increased $44 \%$ with the addition of fermented cactus (T3). Ammonia nitrogen, lactic acid and volatile fatty acids values were different among treatments $(\mathrm{p}<0.05)$. Maximum gas production ( $\mathrm{Gmax}$ ) and $\mathrm{CH} 4$ concentration decreased $32 \%$ and $49 \%$ at T3, respectively. The addition of cactus and fermented cactus to corn stover silage increases protein content. In addition, it reduces ruminal methane synthesis in vitro.
\end{abstract}

Keywords: fermentation, corn stover, greenhouse gases, silage. 


\section{INTRODUCCIÓN}

En el norte de México las temperaturas extremas y la prolongada sequía han causado una disminución en la producción de forrajes. De esta manera, los pequeños productores se han visto en la necesidad de utilizar recursos forrajeros que representan un bajo aporte de nutrientes para los rumiantes para contrarrestar la época de sequías (López-Inzunza et al., 2017). Así, el rastrojo de maíz (Zea mays) ha sido empleado como fuente forrajera en zonas áridas y semiáridas del norte del país como un esquilmo en la producción de granos para consumo humano (SAGARPA, 2009). Bajo estas condiciones de producción, el nopal surge como una alternativa en la alimentación para el ganado (Flores-Hernández et al., 2017). El nopal (Opuntia spp.) es un recurso vegetal importante en el norte de México; se considera un almacén natural de agua y es muy eficiente en el consumo de ésta (Orona-Castillo et al., 2008). Adicionalmente, el nopal proporciona energía digestible, agua y vitaminas al animal durante la época de secas (Dubeux et al., 2018).

También, comparado con otros forrajes anuales, el nopal utiliza menos agua para su producción y crecimiento (Flores-Hernández et al. 2019). Sin embargo, su bajo contenido de proteína (4 \% MS) limita su uso como única fuente de forraje. Debido a lo anterior, se recomienda aplicar diferentes procesos biotecnológicos que ayuden a incrementar su contenido de proteína; por ejemplo, la fermentación en estado sólido (FES) (Herrera et al., 2017). El proceso de FES incrementa el contenido de proteína del sustrato por incremento en la proteína unicelular en la pared celular de los microorganismos. Los microorganismos más utilizados son las levaduras Saccharomyces cerevisiae y algunas especies de Kluyveromyces (Van Markis et al., 2006). Por otro lado, el proceso de ensilaje puede servir para disminuir los problemas de la alimentación del ganado y enfrentar la escasez de forraje en la época seca (Castro et al., 2016). Este proceso inhibe el crecimiento de microorganismos patógenos mediante la disminución del $\mathrm{pH}$, debido a la presencia de bacterias ácido-lácticas (BAL), lo cual permite conservar la frescura y las características nutricionales de los forrajes para su posterior uso (Mokoboki et al., 2016). Debido a lo anterior, el objetivo de este trabajo fue evaluar la calidad nutritiva y la producción de gas in vitro de ensilados de rastrojo de maíz con la adición de nopal y nopal fermentado.

\section{MATERIAL Y MÉTODOS}

\section{Área de estudio}

El estudio se llevó a cabo en la Facultad de Medicina Veterinaria y Zootecnia de la Universidad Juárez del Estado de Durango. El nopal forrajero variedad AV6 fue cosechado al azar de una nopalera cultivada, localizada a un costado de la Facultad y dentro del municipio de Durango, Durango, México.

\section{Fermentación es estado sólido (FES) y preparación de los microsilos}

Las pencas de nopal fueron cortadas en piezas de aproximadamente $1 \mathrm{~cm}^{2}$ utilizando un cuchillo de acero inoxidable y colocadas en contenedores de plástico de 19 I, en donde fueron inoculadas con Sacharomyces cerevisiae $(1 \% \mathrm{~m} / \mathrm{m})$. El proceso de fermentación 
fue llevado a cabo por $48 \mathrm{~h}$ a $25^{\circ} \mathrm{C}$. Los tratamientos consistieron en incluir nopal y nopal fermentado a forraje de maíz, como se muestra en la tabla 1. Microsilos experimentales fueron preparados con forraje de maíz picado sin grano, y en estado maduro con un tamaño de partícula de 2 a $4 \mathrm{~cm}$ (Variedad: hibrído 21/20) (T1, $n=7)$, rastrojo de maíz con nopal fresco $(T 2, n=7)$ y rastrojo de maíz con nopal fermentado ( $T 3, n=7)$ en contenedores de plástico $(30 \mathrm{~cm}$ diámetro $\times 50 \mathrm{~cm}$ alto), sellados herméticamente por $30 \mathrm{~d}$. Después de este tiempo, los microsilos fueron abiertos para su posterior análisis.

Tabla 1. Proporción de los ingredientes en los tratamientos experimentales

\begin{tabular}{lccc}
\hline (\%) & T1 & T2 & T3 \\
\hline Ingredientes & & 75 & 75 \\
Rastrojo de maíz & 100 & 25 & -- \\
Nopal sin fermentar & -- & -- & 25 \\
Nopal fermentado & -- & & \\
\hline
\end{tabular}

\section{Variables fermentativas}

Una vez que fueron abiertos los microsilos se evaluaron las siguientes variables: $\mathrm{pH}$ (Hanna instruments, modelo HI 83142); ácido láctico de acuerdo con Borshchevskaya et al. (2016); así como los contenidos de ácidos grasos volátiles y nitrógeno amoniacal (NH3-N), empleando los procedimientos propuestos por Galyean (2010).

\section{Análisis químico}

Las muestras de cada microsilo experimental fueron secadas en una estufa de aire forzado a $55^{\circ} \mathrm{C}$ por $72 \mathrm{~h}$; posteriormente se redujo el tamaño de partícula a $1 \mathrm{~mm}$ en un molino Wileymil (Arthur H Thomas, Philadelphia, PA, USA), para determinar los contenidos de material seca (MS) (método 934.01). La concentración de proteína cruda (PC) fueron determinadas por la técnica de micro-Kjeldhal (método 920.87), utilizando el factor de conversión (6.25) (AOAC, 2010). La concentración de FDN, FDA fueron obtenidas de acuerdo a los procedimientos propuestos por Van Soest (1991) y los parámetros de producción de gas de acuerdo a la técnica descrita por Menke y Steingass (1988).

\section{Producción de gas in vitro}

Aproximadamente $1 \mathrm{~g}$ de muestra de cada microsilo experimental fue colocado en módulos de vidrio en un equipo transductor de presión marca ANKOM e incubados por triplicado con una solución 2:1 de solución buffer-líquido ruminal, de acuerdo al procedimiento descrito por Murillo-Ortiz et al. (2018). Las incubaciones fueron llevadas a cabo desde las 0 hasta las $96 \mathrm{~h}$ y registrando los valores de presión al mismo tiempo. 
La cinética de producción de gas fue estimada mediante la función de Gompertz (MurilloOrtiz et al., 2018), de acuerdo a la siguiente ecuación:

$$
G P=G \max * \exp [-A * \exp (-k * t)]
$$

Donde $\mathrm{GP}=$ producción de gas al tiempo $\mathrm{t}(\mathrm{ml}), \mathrm{Gmax}=$ producción máxima de gas $(\mathrm{ml})$, $\mathrm{k}=$ tasa constante de producción de gas $\left(\mathrm{h}^{-1}\right)$ y $\mathrm{A}=$ fase lag (h). De la incubación de las $24 \mathrm{~h}$, se abrió la válvula para liberar gas durante $2 \mathrm{~s}$ de cada módulo. El gas liberado de cada módulo se conectó a un analizador portátil de $\mathrm{CH}_{4}$ y $\mathrm{CO}_{2}$ a través de un tubo para medir la concentración de estos gases de acuerdo a los procedimientos establecidos por el fabricante (GEM ${ }^{\mathrm{TM}} 5000$, LANDTEC, USA).

\section{Parámetros de fermentación in vitro}

Para evaluar los parámetros de fermentación, se colocó $1 \mathrm{~g}$ de muestra en bolsas de nylon (ANKOM, F500 nylon bags; ANKOM, 2018), pesadas previamente y colocadas dentro de los módulos ANKOM e incubadas por triplicado con solución buffer:líquido ruminal, en una relación 2:1 de acuerdo con Murillo-Ortiz et al. (2018). Después de $24 \mathrm{~h}$ de fermentación continua, los módulos fueron abiertos e inmediatamente se midió el pH (Hanna instruments, model HI 83142). Las bolsas se sacaron de los módulos y se lavaron con agua destilada y secadas a $65^{\circ} \mathrm{C}$ por $48 \mathrm{~h}$. La digestibilidad in vitro de la materia seca (DIVMS) se calculó en base a la diferencia en el contenido de materia seca del sustrato antes y después de la incubación. Adicionalmente y aproximadamente $1.0 \mathrm{ml}$ del filtrado fueron centrifugados a 3,000×g por $5 \mathrm{~min}$; luego, $500 \mu \mathrm{l}$ del líquido sobrenadante fue acidificado con $150 \mu \mathrm{l}$ de ácido meta fosfórico al $25 \%$ para evaluar ácidos grasos volátiles. También aproximadamente $1.0 \mathrm{ml}$ del filtrado fue colocado en tubos y acidificado con $30 \mu \mathrm{l}$ de ácido sulfúrico al $50 \%$ v/v para determinar $\mathrm{N}-\mathrm{NH}_{3}$ (Galyean, 2010).

\section{Análisis estadístico}

Los datos obtenidos fueron analizados con un diseño completamente al azar, utilizando los procedimientos GLM de SAS (2010). Las medias fueron comparadas con la prueba de rango múltiple de Tukey y declarando diferencias significativas cuando fue $P \leq 0.05$.

\section{RESULTADOS Y DISCUSIÓN}

\section{Composición química}

El contenido de materia seca (MS) disminuyó con la inclusión de rastrojo de maíz $(\mathrm{P}<0.05$, tabla 2). Los ensilados que contienen rastrojo y nopal (t2 y t3) presentaron contenidos de MS adecuados, ya que de acuerdo con Pineda-Cordero (2016) los ensilados que contienen entre el 30 y $35 \%$ de MS se consideran de buena calidad. 
Además, la humedad presente en los ensilados determina el tipo de fermentación que se realizó durante el proceso de ensilado. Otros factores que determinan la calidad de la fermentación son los carbohidratos solubles presentes y la capacidad amortiguadora del forraje empleado (Bernal et al., 2002).

La concentración de proteína cruda (PC) fue diferente entre tratamientos $(\mathrm{P}<0.05$, tabla 2). La adición de nopal y nopal fermentado aumentó $43.54 \%$ y 79 \% la concentración de $\mathrm{PC}$ en los ensilados respectivamente, comparados con t1. Los incrementos en $\mathrm{t} 2$ y t3 se deben al contenido de proteína inicial en el rastrojo de maíz antes de ser ensilado (el forraje verde maduro contenía $4.9 \%$, mientras que el rastrojo contenía $5.2 \%$ de PC; resultados no mostrados) y a la proliferación de proteína celular proveniente de la levadura Saccharomyces cereviseae empleada para fermentar el nopal. No obstante, Alhanafi et al. (2019) registraron un menor contenido de PC en ensilados de Opuntia ficus, indica adicionado con Atriplex (6.41\%); en comparación con t2 de este estudio.

Tabla 2. Composición química de los ensilados de rastrojo de maíz adicionados con nopal

\begin{tabular}{|c|c|c|c|c|}
\hline$(\%)$ & $\mathrm{T} 1$ & $\mathrm{~T} 2$ & T3 & EEM \\
\hline Materia seca & $42.0 \pm 0.29^{a}$ & $35.9 \pm 0.48^{b}$ & $35.5 \pm 0.26^{b}$ & 0.25 \\
\hline Proteína cruda & $6.2 \pm 0.55^{c}$ & $8.9 \pm 0.10^{b}$ & $11.1 \pm 0.05^{\mathrm{a}}$ & 0.05 \\
\hline Fibra detergente neutro & $53.2 \pm 2.31^{\mathrm{b}}$ & $63.1 \pm 0.08^{a}$ & $58.8 \pm 0.12^{\mathrm{ab}}$ & 1.09 \\
\hline Fibra detergente ácida & $23.6 \pm 0.06^{c}$ & $37.6 \pm 0.05^{\mathrm{a}}$ & $35.3 \pm 0.73^{b}$ & 0.34 \\
\hline Digestibilidad de la materia seca & $61.8 \pm 2.44$ & $58.4 \pm 1.98$ & $64.1 \pm 2.20$ & 1.81 \\
\hline
\end{tabular}

La concentración de fibra detergente neutro (FDN) fue menor en el T1 ( $\mathrm{P}<0.05$, tabla 2); se observó un incremento de $18 \%$ en t2. Este aumento se debe a la presencia del rastrojo de maíz, el cual posee una alta cantidad de fibra; sin embargo, parte de la hemicelulosa es hidrolizada durante el proceso de ensilado. En esta etapa las pentosas se liberan y se fermentan en ácido láctico y ácido acético (McDonald et al., 2002).

Igualmente, el contenido de fibra detergente ácida (FDA) mostró el mismo comportamiento; FDA aumentó 59 y $49 \%$ en T2 y T3, respectivamente $(\mathrm{P}<0.05$, Tabla 2). De la misma manera, los incrementos en FDA se le atribuyen a la presencia de rastrojo de maíz en $\mathrm{t} 2$ y t3. No obstante, los contenidos de FDN y FDA se encuentran dentro del rango de forrajes de buena calidad.

\section{Parámetros de fermentación del proceso de ensilaje}

Los valores de $\mathrm{pH}$ fueron mayores en $\mathrm{t} 2 \mathrm{y} \mathrm{t} 3$, con respecto a t1 $(\mathrm{P}<0.05$, tabla 3$)$; sin embargo, todos se encuentran en los valores ideales, lo cual es indicativo de un buen proceso de fermentación y conservación. Cabe mencionar que la velocidad con la que un 
ensilado logra un $\mathrm{pH}<4.0$ garantiza la estabilidad del mismo y reduce la pérdida de nutrientes por fermentaciones secundarias, o bien por contaminación de bacterias y hongos (Ha Vu et al., 2019).

La concentración de nitrógeno amoniacal en los ensilados se incrementó con la inclusión de nopal y nopal fermentado $(\mathrm{P}<0.05$, tabla 3$)$. Este incremento registrado en los ensilados del t2 y t3 pudo haber sido ocasionado por un aumento en los microorganismos que degradan proteína (Berumen et al., 2015; Ruangyote y Metha, 2018), o también el empleo de rastrojo de maíz reduce la cantidad de carbohidratos solubles y por lo tanto aumenta la degradación de proteínas (Herremans et al., 2019). Sin embargo, para clasificar un ensilado como de buena calidad, la concentración de nitrógeno amoniacal máxima debe ser de 7-20 \% del nitrógeno total (Sánchez y García, 2017); por lo cual los ensilados experimentales obtuvieron valores que se encuentran dentro de este rango y por lo tanto indican que se llevó a cabo un adecuado proceso de fermentación.

Tabla 3. Parámetros de fermentación de ensilados de rastrojo de maíz adicionados con nopal

\begin{tabular}{|c|c|c|c|c|}
\hline & T1 & T2 & T3 & EEM \\
\hline $\mathrm{pH}$ & $4.3 \pm 0.05^{b}$ & $4.7 \pm 0.04^{a}$ & $4.7 \pm 0.01^{\mathrm{a}}$ & 0.03 \\
\hline $\mathrm{N}-\mathrm{NH}_{3}(\mathrm{~g} / \mathrm{kg} \mathrm{MS})$ & $1.9 \pm 0.09^{c}$ & $5.5 \pm 0.09^{b}$ & $6.5 \pm 0.20^{a}$ & 0.03 \\
\hline Ácido Láctico (g/kg MS) & $24.3 \pm 3.45^{b}$ & $73.9 \pm 1.91^{\mathrm{a}}$ & $76.7 \pm 4.18^{\mathrm{a}}$ & 2.71 \\
\hline Ácido Acético (\% MS) & $0.7 \pm 0.26^{c}$ & $0.9 \pm 0.00^{b}$ & $1.1 \pm 0.02^{\mathrm{a}}$ & 0.01 \\
\hline Ácido Propiónico (\% MS) & $3.5 \pm 0.01^{c}$ & $4.2 \pm 0.02^{b}$ & $4.3 \pm 0.01^{a}$ & 0.01 \\
\hline Ácido Butírico (\% MS) & $0.01 \pm 0.002^{b}$ & $0.03 \pm 0.004^{\mathrm{a}}$ & $0.04 \pm 0.001^{a}$ & 0.002 \\
\hline
\end{tabular}

La concentración de ácido acético fue diferente entre tratamientos $(P<0.05$, tabla 3$)$. Los ácidos grasos volátiles son producto de fermentaciones inducidas por la presencia de bacterias coliformes que transforman el ácido láctico en acético y butírico, y que están presentes en el estiércol y la tierra. De acuerdo con Kung et al. (2018), las concentraciones de ácido acético se encuentran entre 0.5 y $2.0 \%$, cuando el contenido de MS es de 45 a $55 \%$; por lo que los valores obtenidos en este trabajo se encuentran dentro del rango para ensilados de buena calidad (0.5-1.1\%). Los valores de ácido propiónico fueron diferentes entre tratamientos $(P<0.05$, tabla 4). Los resultados obtenidos en este estudio son iguales a los reportados por González et al., (2019) en ensilado de maíz con nopal y nopal fermentado (4.0\%). Las concentraciones de ácido butírico fueron mayores en los ensilados que contienen rastrojo $(P<0.05$, tabla 3$)$; sin embargo, estos valores indican que hubo una adecuada fermentación. Además, Da Silva et al. (2020) reporta valores más bajos de ácido propiónico en ensilados de nopal con gliricidia. De acuerdo a los valores obtenidos de ácido láctico y butírico en los ensilados 
de este estudio, se puede inferir que la inclusión de nopal y nopal fermentado en el rastrojo promueve un incremento en el ácido láctico y una disminución en el butírico, lo cual da como resultado ensilados con buena calidad fermentativa y nutrimental.

\section{Parámetros de fermentación ruminal}

La concentración de $\mathrm{N}-\mathrm{NH}_{3}$ fue menor en $\mathrm{t}$, comparado con t2 y $\mathrm{t} 3(\mathrm{P}<0.05$, tabla 4). La inclusión de nopal y nopal fermentado en los ensilados de rastrojo promovió un incremento de $48 \%$ y $27.7 \%$ en $\mathrm{N}^{-N_{3}}$, respectivamente. Cambios en esta variable indican que se está llevando una proteólisis y la cual se incrementó debido al incremento en la proteína cruda en el ensilado por la adición de rastrojo y nopal fermentado. No obstante, hay estudios que afirman que incrementos en la concentración de $\mathrm{N}_{-} \mathrm{NH}_{3}$ se deben a que la proteína no es incorporada a la síntesis de proteína microbiana, lo cual reflejaría una pérdida de energía en el rumiante (Rodríguez et al., 2007). Por el contrario, otros autores también encontraron incrementos en la cantidad de $\mathrm{N}-\mathrm{NH}_{3}$ cuando se incrementó el contenido de proteína cruda a través de la adición de urea en ensilados de piña (López-Herrera et al., 2014). Adicionalmente, estos resultados coinciden con los obtenidos por Pinho et al. (2017) en ensilados de nopal (17 mg/dL) a las 9 horas de incubación. De tal manera, en este estudio las concentraciones de $\mathrm{N}-\mathrm{NH}_{3}$ registradas en todos los tratamientos presentaron niveles adecuados mayores a $5 \mathrm{mg} / \mathrm{dL}$, lo que permite garantizar la síntesis de proteína microbiana (Rodríguez et al., 2007).

Tabla 4. Parámetros de fermentación ruminal in vitro de ensilados de rastrojo de maíz adicionados con nopal

\begin{tabular}{lcccc}
\hline & T1 & T2 & T3 & EEM \\
\hline $\mathrm{pH}$ & $6.8 \pm 0.008$ & $6.9 \pm 0.17$ & $6.9 \pm 0.03$ & 0.01 \\
$\mathrm{~N}-\mathrm{NH}_{3}(\mathrm{mg} / \mathrm{dL})$ & $11.9 \pm 1.08^{\mathrm{b}}$ & $17.7 \pm 0.43^{\mathrm{a}}$ & $15.2 \pm 0.08^{\mathrm{a}}$ & 0.55 \\
Ácido Acético (\%) & $53.3 \pm 0.89^{\mathrm{a}}$ & $52.1 \pm 0.48^{\mathrm{a}}$ & $46.5 \pm 0.43^{\mathrm{b}}$ & 0.52 \\
Ácido Propiónico (\%) & $27.1 \pm 0.72^{\mathrm{b}}$ & $30.6 \pm 0.31^{\mathrm{a}}$ & $32.1 \pm 0.45^{\mathrm{a}}$ & 0.43 \\
Ácido Butírico (\%) & $14.7 \pm 0.05^{\mathrm{a}}$ & $12.6 \pm 0.13^{\mathrm{b}}$ & $14.7 \pm 0.05^{\mathrm{a}}$ & 0.07 \\
\hline a,b Letras diferentes en la misma fila indican diferencias $(\mathrm{P}<0.05)$. EEM=error estándar de la media. $\mathrm{N}-\mathrm{NH}_{3}$ nitrógeno amoniacal; $\mathrm{n}=3$.
\end{tabular}

Por otro lado, la concentración de ácido acético disminuyó y la de propionato se incrementó en los ensilados de rastrojo de maíz y nopal fermentado $(P<0.05$; tabla 4$)$, en comparación con 11 y t2. En este sentido, un decremento en la concentración de ácido acético en $\mathrm{t} 2$ y $\mathrm{t} 3$, se encuentra estrechamente relacionado con una disminución en la fermentación de carbohidratos estructurales (FDN y FDA). De esta manera, al ser los carbohidratos estructurales los de mayor concentración en los nutrientes de $\mathrm{t} 2$ y t3, se sugiere que no se está llevando una fermentación adecuada (Van Soest, 1994). Sin 
embargo, Sánchez et al. (2014) también detectaron una disminución en la concentración de acetato y un incremento en el propionato ruminal. Regularmente, la tasa de producción de propionato y otros AGV está directamente relacionada con el consumo de sustratos fermentables de la dieta, lo que favorece la síntesis de propionato a partir de la fermentación microbiana por las bacterias amilolíticas (Van Soest, 1994).

La máxima producción de gas (Gmax) fue mayor en t1 ( $\mathrm{P}<0.05$; tabla 5). La inclusión de nopal y nopal fermentado en ensilados con rastrojo de maíz disminuyó la máxima producción de gas $31.9 \%$ y $48.7 \%$, respectivamente. Los valores obtenidos en este estudio son menores a los reportados en leucaena y pasto estrella $(234$ y $154 \mathrm{ml} / \mathrm{g}$, respectivamente) por Naranjo et al. (2016). De igual forma, González et al. (2019) registraron valores de Gmax mayores en ensilados de maíz con nopal (176 ml). La disminución en la producción de gas observada en este estudio se le atribuye a la disminución de la digestibilidad de la materia seca en $\mathrm{t} 2$ y t3, como resultado de la adición de rastrojo de maíz, cuyos valores de FDN y FDA son más altos que los registrados en t1. Así como se expresó anteriormente, la presencia de carbohidratos estructurales impiden que se lleve una fermentación adecuada, lo cual se ve reflejado en la producción de gas.

Tabla 5. Parámetros de cinética de gas de los ensilados de rastrojo de maíz adicionados con nopal

\begin{tabular}{|c|c|c|c|c|}
\hline Parámetro & T1 & $\mathrm{T} 2$ & T3 & EEM \\
\hline Gmax (ml/g MS) & $124.8 \pm 8.48^{\mathrm{a}}$ & $94.6 \pm 6.50^{b}$ & $84.0 \pm 1.75^{b}$ & 5.10 \\
\hline$A(h)$ & $4.2 \pm 0.21^{a}$ & $2.7 \pm 0.03^{b}$ & $4.4 \pm 0.04^{a}$ & 0.10 \\
\hline $\mathrm{K}(\% / \mathrm{h})$ & $0.08 \pm 0.001$ & $0.07 \pm 0.007$ & $0.09 \pm 0.003$ & 0.003 \\
\hline Metano (ml/g MS) & $9.7 \pm 0.29^{a}$ & $7.3 \pm 0.29^{b}$ & $6.5 \pm 0.16^{b}$ & 0.21 \\
\hline $\mathrm{CO}_{2}(\mathrm{ml} / \mathrm{g} \mathrm{MS})$ & $59.0 \pm 4.49^{a}$ & $49.8 \pm 0.75^{\mathrm{ab}}$ & $44.7 \pm 0.70^{\mathrm{b}}$ & 2.17 \\
\hline Relación metano: $\mathrm{CO}_{2}$ & $0.16 \pm 0.010^{a}$ & $0.14 \pm 0.003^{b}$ & $0.14 \pm 0.005^{b}$ & 0.014 \\
\hline
\end{tabular}

Por su lado, el periodo de latencia "A" disminuyó $55 \%$ en el t2 (ensilado de rastrojo + nopal). Para explicar este resultado se deben tomar en cuenta variables que no fueron consideradas en este estudio, como el contenido de carbohidratos solubles o la lignina; ya que de estos depende que el inicio de la fermentación sea más rápido. En este sentido, López-Inzunza et al. (2017) reportaron mayores tiempos de latencia para ensilados con mayor contenido de FDA, con respecto al contenido de FDN en ensilados con rastrojo de piña; estos autores mostraron concentraciones de carbohidratos estructurales y tiempos de latencia similares a los reportados en este estudio (72\% de FDN y una A de $3.8 \mathrm{~h}$ ). 
Además, los valores de A obtenidos en este estudio también son similares a los registrados por González et al. (2019) en ensilados de forraje de maíz con nopal. Adicionalmente, la producción de metano disminuyó 32 y $49 \%$ en los ensilados que incluyeron nopal y nopal fermentado, respectivamente $(\mathrm{P}<0.05$; tabla 5$)$. De la misma manera, la relación metano: $\mathrm{CO}_{2}$ también disminuyó con la adición de nopal y nopal fermentado, así como de rastrojo de maíz. Esta variable está estrechamente relacionada con la síntesis de metano ruminal. Mayores valores en esta relación sugiere un incremento en la síntesis de metano ruminal a través de la ruta de la reducción de $\mathrm{CO}_{2}$ (Murillo et al., 2018). De esta manera, aunque una reducción en la producción de metano y $\mathrm{CO}_{2}$ están estrechamente relacionados con una disminución en la calidad fermentativa de los ensilados $t 2$ y $\mathrm{t} 3$, esta reducción también sugiere cambios o incluso una inhibición en las poblaciones metanogénicas (Tavendale et al., 2005). Además, la disminución de metano está directamente relacionada con un aumento en la producción de propionato ruminal, tal y como lo muestra el presente estudio.

\section{CONCLUSIONES}

La adición de nopal y nopal fermentado a ensilados con rastrojo de maíz incrementa el contenido de proteína cruda 43 y $79 \%$, respectivamente. Además, el uso de nopal y nopal fermentado en ensilados de rastrojo de maíz reduce la síntesis de metano ruminal in vitro. Por el contrario, la presencia de rastrojo de maíz incrementa los contenidos de carbohidratos estructurales en el ensilado, lo cual compromete la fermentacion ruminal y la producción de gas in vitro; sin embargo, se recomienda llevar a cabo estudios in vivo que confirmen estos resultados.

\section{LITERATURA CITADA}

ALHANAFI F, Kaysi Y, Muna M, Alkhtib A, Wamatu J, Burton E. 2019. Spineless cactus (Opuntia ficus-indica) and saltbush (Atriplex halimus L.) as feed supplements for fattening Awassi male lambs: effect on digestibility, water consumption, blood metabolites, and growth performance. Tropical Animal Health and Production. 51:1637-1644. https://doi.org/10.1007/s11250-019-01858-6

ANKOM. 2018. RF Gas production system operator's manual. ANKOM Technology, USA. https://www.ankom.com/sites/default/files/document-files/RF_Manual.pdf

AOAC. 2010. Official Methods of Analysis. Association of Official Analytical Chemists International. Gaithersburg, Maryland. 18 ed. 3er. revisión. 2590 Pp. ISBN: 9780935584820

BERNAL J, Chaverra H, Arciniegas A, Acevedeo G, Angel M. 2002. Ensilaje, Heno y Henolaje. $1^{\underline{a}}$ edición, Editorial Angel Comunicaciones. Bogotá, Colombia. ISBN: 978-95844-1174-7 
BERUMEN HL, Páez J, Soto NO, Murillo M, Herrera E, Muro A. 2015. Chemical composition, in vitro gas production and energy value of prickly pear fermented with and without Kluyveromyces marxianus. Journal of BioScience and Biotechnology. 4(3):359364.

https://www.ingentaconnect.com/content/doaj/13146238/2015/00000004/00000003/art0 0014

BORSHCHEVSKAYA LN, Gordeeva TL, Kalinina AN, Sineokii SP. 2016. Spectrophotometric determination of lactic acid. Journal of Analytical Chemistry. 71:755758. https://doi.org/10.1134/s1061934816080037

CASTRO-RINCÓN E, Sierra-Alarcón A, Mojica-Rodríguez J, Carulla-Fornaguera J, Lascano-Aguilar C. 2016. Uso múltiple de leguminosas como abono verde, en rotación con maíz, y heno, para producción de leche. Corpoica Ciencia y Tecnología Agropecuaria. 17(1):17-29. https://doi.org/10.21930/rcta.vol20_num3_art:1586

DA SILVA-BRITO GM, Santos EM, García G, Silva de Oliveira J, Zanine AM, Alexandre Fernandes-Perazzo A, Sena-Campos F, Vasconcelos de Oliveira AG, Cavalcanti HS. 2020. Mixed silages of cactus pear and gliricidia: chemical composition, fermentation characteristics, microbial population and aerobic stability. Scientific Reports. Nature research. 10:6834. https://doi.org/10.1038/s41598-020-63905-9

DUBEUX JCB, Ben Salem H, Nefzaoui Al. 2018. Producción y utilización del nopal forrajero en la nutrición animal. En: Ingles PC, Mondragon J, Nefzaoui A y Sáenz C. (ed.). Ecología del cultivo, manejo y usos del nopal. FAO-ICARDA. Pp. 229. ISBN 978-92-5130494-5. http://www.fao.org/3/i7628es/l7628ES.pdf

FLORES-HERNÁNDEZ A, Araújo-Filho JT, Gomes da Silva F, Ramírez-Ordoñez S, Murillo-Amador B. 2017. Dietas a base de forraje tradicional y nopal (Opuntia spp.) enriquecido con proteínas para alimentar cabras. Nova Scientia. 9(18): 149-166. https://doi.org/10.21640/ns.v9i18.828

FLORES-HERNÁNDEZ A, Macías-Rodríguez FJ, Meza-Herrera C, García-Herrera G, Esquivel-Arriaga O, Ortiz-Salazar J, Hernández-Bautista C. 2019. Semi-solid fermentation of nopal (Opuntia spp) for use as an animal protein supplement. Revista de Geografía Agrícola. 63: 87-100. https://doi.org/10.5154/r.rga.2019.63.04

GALYEAN ML. 2010. Laboratory Procedures for Animal Nutrition Research, $14^{\text {th }}$ edn. Department of Animal and Food Sciences, Texas Tech University, Lubbock, Texas. https://www.dpts.ttu.edu/afs/home/mgalyean/lab_man.pdf 
GONZÁLEZ AA, Murillo OM, Pámanes CG, Reveles SF, Herrera TE. 2019. Nutritive quality and gas production of corn silage with the addition of fresh and fermented prickly pear cladodes. Journal of Animal and Plant Sciences. 40(1): 6544-6553. https://m.elewa.org/Journals/wpcontent/uploads/2019/04/4.Gonzalez.pdf

HA VU V, Li X, Wang M, Liu R, Zhang G, Liu W, Xia B, Sun Q. 2019. Dynamics of fungal community during silage fermentation of elephant grass (Pennisetum purpureum) produced in northern Vietnam. Asian-Australasian Journal of Animal Science. 32(7):9961006. https://doi.org/10.5713/ajas.18.0708

HERREMANS S, Decruyenaere V, Beckers Y, Froidmont E. 2019. Aditivos de ensilaje para reducir la degradación de proteínas durante el ensilado y evaluación de la degradabilidad del nitrógeno ruminal in vitro. Grass and forage sciences. 74(1):86-96. https://doi.org/10.1111/gfs.12396

HERRERA TE, Murillo M, Berumen L, Soto-Cruz NO, Páez-Lerma JB. 2017. Protein enrichment of Opuntia Ficus-indica using Kluyveromyces marxianus in solid-state fermentation. Ciencia e Investigación Agraria. 44:113-120. https://doi.org/10.7764/rcia.v44i2.1767

KUNG L, Shaver RD, Grant, RJ, Schmidt RJ. 2017. Silage review: Interpretation of chemical, microbial, and organoleptic components of silages. Journal of Dairy Science. 101: 4020-4033. https://doi.org/10.3168/jds.2017-13909

LÓPEZ-HERRERA M, Jones RWC, Rojas-Bourrillón A, Rodríguez-Chacón S. 2014. Valor nutricional del ensilaje de rastrojo de piña con niveles crecientes de urea. Nutrición Animal Tropical. 8(1): 1-20. https://revistas.ucr.ac.cr/index.php/nutrianimal/article/view/14989

LÓPEZ-INZUNZA HJ, Chongo-García BB, O-León OL, Guerra-Liera JE, Luna-López M, Castro-Camacho SJ, López-Juárez LA. 2017. Digestibilidad in situ de rastrojo de maíz tratado con enzimas fibrolíticas. Revista Ciencia y Agricultura. 14(1): 31-37. http://www.doi.org/10.19053/01228420.v14.n1.2017.6085

MCDONALD P, Edwards RA, Greenhalgh JF. 2002. Animal Nutrition. 6th Edition. Longman, London and New York. Pp. 543. ISBN-10:9781408204238

MENKE KH, Steingass H. 1988. Estimation of the energetic feed value obtained from chemical analysis and in vitro gas production using rumen fluid. Animal Research and Development. 28:7-55. https://www.scienceopen.com/document?vid=e1859372-e696424a-85fb-d305b0b594bc

MOKOBOKI K, Sebola N, Matlabe G. 2016. Efecto de los niveles de melaza y las condiciones de crecimiento sobre el valor nutritivo y la calidad de fermentación de ensilaje de cladodios de Opuntia. Journal of Animal Plant Science. 28: 4488-4495. https://doi.org/10.4141/cjas2013-188 
MURILLO OM, Herrera TE, Corral LA, Pámanes CG. 2018. Effect of inclusion of graded level of water hyacinth on in vitro gas production kinetics and chemical composition of alfalfa hay based beef cattle diets. Indian Journal of Animal and Research. 52(8): 12981303. https://doi.org/10.18805/ijar.11417

NARANJO JF, Ceballos OA, Gaviria X, Tarazona AM, Correa A, Chará JD, Murgueitio E, Barahona R. 2016. Estudio de la cinética fermentativa in vitro de mezclas de forrajes que incluyen Leucaena leucocephala proveniente de sistemas silvopastoriles intensivos (SSPi) en Colombia. Revista CES Medicina Veterinaria y Zootecnia. 11(2): 6-17. https://doi.org/10.21615/cesmvz.11.2.1

ORONA-CASTILLO I, Flores-Hernández A, Rivera GM. 2008. Manual para el establecimiento y manejo del nopal verdura bajo riego por goteo en la Comarca Lagunera. CENID RASPAINIFAP, Gómez Palacio, Durango, México. Pp.18.

http://biblioteca2.uaaan.mx/cgi-bin/koha/opac-detail.pl?biblionumber=4441

PINEDA-CORDERO L, Chacón-Hernández P, Boschini-Figueroa C. 2016. Evaluación de la calidad del ensilado de pasto estrella africana (Cynodon nlemfluensis) mezclado con tres diferentes aditivos. Agronomía Costarricense. 40(1) 1:11-27. http://www.redalyc.org/articulo.oa?id=43646210001

PINHO RM, Santos EM, Oliveira, JS, Loureiro, AHR, Macêdo, AJS, Alves JP, Santos AP, Santos VS. 2017. Effect of spineless-cactus mucilage on the in vitro rumen fermentation of cellulose, starch, and protein. Revista Brasileira Saúde e Produção Animal. 18(4):505517. https://doi.org/10.1590/s1519-99402017000400002

RODRÍGUEZ R, Sosa A, Rodríguez Y. 2007. Microbial protein synthesis in rumen and its importance to ruminants. Cuban Journal of Agricultural Science. 41(4): 287-294. https://www.researchgate.net/profile/Rafael-Rodriguez-

10/publication/276901672_Microbial_protein_synthesis_in_rumen_and_its_importance_ to_ruminants/links/555b266908aeaaff3bfbcad0/Microbial-protein-synthesis-in-rumenand-its-importance-to-ruminants.pdf

SAGARPA. 2009. Aprovechamiento de esquilmos y subproductos en la alimentación de ganado. [Consulta: 2 de Marzo de 2021]. Disponible en:

https://www.academia.edu/19017564/Aprovechamiento_de_esquilmos

SÁNCHEZ-DUARTE, Garcia A. 2017. Ammonia-N concentration in alfalfa silage and its effects on dairy cow performance: A meta-analysis. Revista Colombiana de Ciencias Pecuarias. 30(3):175-184. https://doi.org/10.17533/udea.rccp.v30n3a01 
SANCHEZ PH, Tracey LN, Browne-Silva J, Lodge-Ivey SL. 2014. Propionibacterium acidipropionici P169 and glucogenic precursors improve rumen fermentation of lowquality forage in beef cattle. Journal of Animal Science. 92:1738-1746. https://doi.org/10.2527/jas.2013-7148

SAS Institute. 2010. Statistical Analysis Software SAS/STAT®. version 9.0.2, Cary, N.C., USA: SAS Institute Inc., ISBN: 978-1-60764-599-3.

http://www.sas.com/en_us/software/analytics/stat.html\#

TAVENDALE MH, Meagher LP, Pacheco D, Walker N, Attwood GT, Sivakumaran S. 2005. Methane production from in vitro rumen incubations with Lotus pedunculatus and Medicago sativa, and effects of extractable condensed tannin fractions on methanogenesis. Animal Feed Science and Technology.123-124(1): 403-419. https://doi.org/10.1016/j.anifeedsci.2005.04.037

VAN MARKIS AJA, Abbot DA Bellissimi E. 2006. Alcoholic fermentation of carbon sources in biomass hy-drolysates by Saccharomyces cerevisiae: current status. Antonie Van Leewenhoek. 90: 391-418. https://doi.org/10.1007/s10482-006-9085-7

VAN SOEST PJ, Robertson JB, Lewis BA. 1991. Methods for dietary fiber, neutral detergent fiber and non-starch polysaccharides in relation to animal nutrition: carbohydrate methodology, metabolism and nutritional implications in dairy cattle. Journal of Dairy Science. 74:35-83. https://doi.org/10.3168/jds.s0022-0302(91)78551-2

VAN SOEST PJ. 1994. Nutritional ecology of the ruminant. 2nd Edition, Cornell University Press, Ithaca, USA. Pp. 476. ISBN: 080142772X. 